\title{
Development and characterization of a new swine model of invasive pneumococcal pneumonia
}

\author{
Rosanel Amaro $1,2,3,17$, Gianluigi Li Bassi ${ }^{1,2,4,5,17}$, Ana Motos ${ }^{1,2,3,6,17}$, Laia Fernandez-Barat $\mathbb{B}^{1,2,3,6}$,

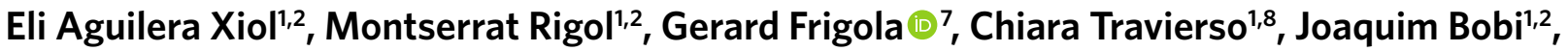 \\ Francesco Pagliara9,10, Marco Carbonara ${ }^{1,11}$, Talitha Comaru ${ }^{1012}$, Chiara Chiurazzi ${ }^{1,13}$, Minlan Yang ${ }^{1,2}$, \\ Hua Yang, 1,2, Marta Arrieta', Joan Daniel Marti', Francesca De Rosa', Maria Adela Saco7, \\ Mariano Rinaudo1, Silvia Terraneo', Marcus J. Schultz ${ }^{14}$, David P. Nicolau ${ }^{15}$, Antonio Artigas ${ }^{3,16}$, \\ Jose Ramirez ${ }^{7}$ and Antoni Torres $1 \mathbb{1}^{1,2,3,6 \bowtie}$
}

\begin{abstract}
Streptococcus pneumoniae is the most common microbial cause of community-acquired pneumonia. Currently, there are no available models of severe pneumococcal pneumonia in mechanically ventilated animals to mimic clinical conditions of critically ill patients. We studied endogenous pulmonary flora in $\mathbf{4}$ healthy pigs and in an additional 10 pigs in which we intra-bronchially instilled S. pneumoniae serotype 19 A, characterized by its resistance to penicillin, macrolides and tetracyclines. The pigs underwent ventilation for $72 \mathrm{~h}$. All pigs that were not challenged with S. pneumoniae completed the $72-\mathrm{h}$ study, whereas $30 \%$ of infected pigs did not. At $24 \mathrm{~h}$, we clinically confirmed pneumonia in the infected pigs; upon necropsy, we sampled lung tissue for microbiological/histological confirmation of pneumococcal pneumonia. In control pigs, Streptococcus suis and Staphylococcus aureus were the most commonly encountered pathogens, and their lung tissue mean \pm s.e.m. concentration was $7.94 \pm 20$ c.f.u./g. In infected pigs, S. pneumoniae was found in the lungs of all pigs (mean \pm s.e.m. pulmonary concentration of $1.26 \times 10^{5}$ $\pm 2 \times 10^{2}$ c.f.u./g). Bacteremia was found in $50 \%$ of infected pigs. Pneumococcal pneumonia was confirmed in all infected pigs at $24 \mathrm{~h}$. Pneumonia was associated with thrombocytopenia, an increase in prothrombin time, cardiac output and vasopressor dependency index and a decrease in systemic vascular resistance. Upon necropsy, microbiological/histological pneumococcal pneumonia was confirmed in 8 of 10 pigs. We have therefore developed a novel model of penicillin- and macrolide-resistant pneumococcal pneumonia in mechanically ventilated pigs with bacteremia and severe hemodynamic compromise. The model could prove valuable for appraising the pathogenesis of pneumococcal pneumonia, the effects associated with macrolide resistance and the outcomes related to the use of new diagnostic strategies and antibiotic or complementary therapies.
\end{abstract}

Sisting treptococcus pneumoniae is the most common causative pathogen of community-acquired pneumonia (CAP), inducing highly severe infections and often resulting in unfavorable outcomes. Approximately one-third of hospitalized patients with pneumococcal pneumonia require admission to the intensive care unit $(\mathrm{ICU})^{1}$.

In the last decade, ventilatory management and therapy of these patients have improved, yet pneumococcal CAP remains associated with high mortality ${ }^{1,2}$, particularly in patients with septic shock or those who require mechanical ventilation ${ }^{3}$. In more recent years, macrolide resistance in $S$. pneumoniae has increased substantially. In several regions worldwide, macrolide-resistant pneumococci have become even more common than penicillin-resistant S. pneumoniae ${ }^{4}$, particularly after the introduction of 7 -valent or 13 -valent pneumococcal conjugate vaccines and the rapid decline of penicillin-resistant strains ${ }^{5}$.
Among several S. pneumoniae virulence factors, the capsule is critical for the development of infection. Based on variations of the capsule molecular structure ${ }^{6}$, a total of 93 S. pneumoniae serotypes have been characterized; however, only roughly 20 serotypes can cause human infections. Per epidemiology data, serotypes 1, 4, 5, $7 \mathrm{~F}, 8,12 \mathrm{~F}, 14,18 \mathrm{C}$ and $19 \mathrm{~A}$ are more likely to cause invasive pneumococcal disease, as defined by isolation of $S$. pneumoniae from a normally sterile body site (e.g., blood) ${ }^{6}$. These serotypes comprise the most recent pneumococcal conjugate vaccine ${ }^{7}$. Furthermore, the Spanish National Institute of Health has shown that the most common invasive serotypes in adults included 3,19A and $1^{8}$.

Given the pivotal role of $S$. pneumoniae in $\mathrm{CAP}^{9}$, several animal models have been developed to better understand the pathogenesis of pneumococcal disease and evaluate novel, preventive and therapeutic strategies. Specifically, mice, rats and rabbits ${ }^{10}$ have gained increasing interest for studying $S$. pneumoniae disease. A main

'Division of Animal Experimentation, Pneumology Department, Hospital Clinic, Barcelona, Spain. 'Institut d'Investigacions Biomèdiques August Pi i Sunyer (IDIBAPS), Barcelona, Spain. ${ }^{3}$ Centro de Investigación Biomedica En Red-Enfermedades Respiratorias (CIBERES), Barcelona, Spain. ${ }^{4}$ Critical Care Research Group, Prince Charles Hospital, Chermside, Australia. ${ }^{5}$ University of Queensland, Brisbane, Australia. ${ }^{6}$ University of Barcelona, Barcelona, Spain. ${ }^{7}$ Department of Pathology, Hospital Clinic, Barcelona, Spain. ${ }^{8}$ Division of Pneumology, ASST Rhodense 'Guido Salvini' Hospital, Garbagnate Milanese, Italy. 'Department of Surgical Sciences and Integrated Diagnostics (DISC), IRCCS AOU San Martino IST, Genova, Italy. ${ }^{10}$ San Martino Policlinico Hospital, IRCCS for Oncology and Neurosciences, Genoa, Italy. "University of Milan, Milan, Italy. ${ }^{12}$ Instituto Federal Farroupilha, Santo Ângelo, Brasil (sponsored by CNPq), Santo Ângelo, Brazil. ${ }^{13}$ Humanitas Clinical and Research Center, Rozzano, Milan, Italy. ${ }^{14}$ Department of Intensive Care, Academic Medical Center, University of Amsterdam, Amsterdam, Netherlands. ${ }^{15}$ Center for Anti-Infective Research \& Development, Hartford Hospital, Hartford, CT, USA. ${ }^{16}$ Pathophysiological Laboratory, Institut de Investigacion Parc Tauli, Corporació Sanitaria Universitaria Parc Tauli, Autonomous University of Barcelona, Sabadell, Barcelona, Spain. ${ }^{17}$ These authors contributed equally: Rosanel Amaro, Gianluigi Li Bassi, Ana Motos. ${ }^{凶}$-mail: atorres@clinic.cat 
limitation of these small animal models is the difficulty in reproducing the severity of human disease or prolonged mechanical ventilation. Severe infections kill small animals quickly and, as a result, prevent investigators from performing a comprehensive assessment of disease dynamics. To date, no reliable model of severe pneumococcal pneumonia reproducing intensive care settings is available. However, in the last decade, our group has developed several other models of respiratory infections caused by nosocomial pathogens, that is, Pseudomonas aeruginosa and methicillin-resistant Staphylococcus aureus ${ }^{11,12}$.

We now describe a pioneering model of pneumococcal pneumonia caused by penicillin- and macrolide-resistant $S$. pneumoniae serotype $19 \mathrm{~A}$ in mechanically ventilated pigs. We fully characterize the infection, focusing on the dynamics of clinical variables, pulmonary mechanics, hemodynamics, inflammatory markers and microbiology studies. Furthermore, using this model and as a proof of concept, we aim to describe the pharmacokinetics of ceftriaxone and levofloxacin in pigs, two first-line antibiotics for pneumococcal pneumonia.

\section{Results}

All pigs not challenged with $S$. pneumoniae completed the study ( $n=4 / 4$ pigs, $100 \%$ ). Conversely, $76-\mathrm{h}$ survival in pigs infected with $S$. pneumoniae was $70 \%(n=7 / 10$ pigs $)$, because two pigs were euthanized at 34 and $48 \mathrm{~h}$, respectively, for severe respiratory/ hemodynamic instability, and one pig was euthanized at $55 \mathrm{~h}$ due to severe hemodynamic instability.

Tracheal secretions, bronchoalveolar lavage and blood cultures. Tracheal secretions were collected at baseline, 24, 48 and $72 \mathrm{~h}$ in both the preliminary study (control pigs) and the main study (pigs infected with S. pneumonia) (Fig. 1). In control pigs, tracheal secretions at baseline were obtained in only one pig, and those were colonized by Bordetella bronchiseptica. At 24, 48 and $72 \mathrm{~h}$, all tracheal secretions in control pigs were colonized by at least one pathogen ( $n=12$ samples) (Fig. 2). In infected pigs, five samples, which were colonized by endogenous flora, were obtained at baseline. By contrast, at 24 and $48 \mathrm{~h}$, all tracheal aspirates in infected pigs were colonized by $S$. pneumoniae at a concentration $>10^{6}$ c.f.u. $/ \mathrm{ml}(n=19$ samples $)$; whereas, at $72 \mathrm{~h}$, six out of seven (86\%) tracheal aspirates were colonized by $S$. pneumoniae at a concentration $>10^{6}$ c.f.u. $/ \mathrm{ml}$ (Fig. 2). The mean $S$. pneumoniae concentration across study measures (i.e., from 24 to $72 \mathrm{~h}$ ) was $6.31 \times 10^{7}$ c.f.u. $/ \mathrm{ml}$, vastly overtaking all other pathogen concentrations. Importantly, at $24 \mathrm{~h}$, pneumococcal pneumonia was clinically confirmed in all infected pigs by $\geq 10^{6}$ c.f.u./ml in tracheal secretions, plus at least one clinical feature. Pathogens in tracheal secretions varied between infected and control pigs, specifically at $24 \mathrm{~h}$ (Fig. 2). As expected, $S$. pneumoniae was consistently the most frequent pathogen in infected pigs at each assessment time point. Conversely, Streptococcus suis, S. aureus and B. bronchiseptica were the most frequently isolated pathogens in control pigs.

Bronchoalveolar lavage (BAL) was performed to quantify bacterial burden at baseline and at 24 and $72 \mathrm{~h}$. In control pigs, colonization occurred in $50 \%(n=2$ out of 4$)$ of BAL samples at baseline. However, at 24 and $72 \mathrm{~h}$, colonization occurred in $100 \%$ of BAL samples ( $n=4$ samples per time point) (Fig. 3 ). In infected pigs, 6 out of $10(60 \%)$ BAL samples were colonized at baseline. After bacterial challenge (at the 24-h time point), the S. pneumoniae concentration significantly increased up to $1.20 \times 10^{6}$ c.f.u./ml. All infected pigs presented with an $S$. pneumoniae concentration $>10^{3}$ c.f.u. $/ \mathrm{ml}$ in BAL fluids at $24 \mathrm{~h}$ ( $n=10$ samples); however, at the end of the study, only $86 \%$ of these pigs presented with the aforementioned S. pneumoniae concentration ( $n=6$ out of 7 samples). Pathogens varied between infected or control pigs at $72 \mathrm{~h}(P=0.04)$ (Fig. 3). Concomitant pathogens progressively increased in infected pigs throughout the study time $(P=0.02)$, yet not notably in control pigs $(P=0.08)$.

Finally, of the 35 assayed blood samples from infected pigs (10 samples at baseline, 10 at $24 \mathrm{~h}, 8$ at $48 \mathrm{~h}$ and 7 at $72 \mathrm{~h}), 8(22.8 \%)$ tested positive for $S$. pneumoniae. $S$. pneumoniae bacteremia was found in five $(50 \%)$ infected pigs during the experiment; specifically, one pig tested positive at 24 and $48 \mathrm{~h}$, two pigs at $48 \mathrm{~h}$ and two pigs at 48 and $72 \mathrm{~h}$. Conversely, controls never developed bacteremia ( $n=0$ out of 16 samples) ( $P=0.045$ versus infected pigs).

Pulmonary tissue burden. Macroscopic findings were observed in lungs upon necropsy (Supplementary Fig. 1). Histological features of infected pigs corroborated confluent pneumonia in all lung tissue samples (Fig. 4). S. pneumoniae was the most commonly isolated pulmonary pathogen ( $n=43$ out of 50 samples; $86 \%$ ) in the infected group. The mean \pm s.e.m. pulmonary $S$. pneumoniae concentration among the 10 infected pigs was $1.26 \times 10^{5} \pm 2 \times 10^{2}$ c.f.u./g, and it did not differ among the 50 sampled lobes (i.e., five lobes/pig; $P=0.92$ ) (Fig. 5). Fifteen of fifty (30\%) analyzed lobes in infected pigs presented concomitant colonization by other pathogens. Nevertheless, the mean \pm s.e.m. concentration of these other pathogens among 50 sampled lobes was 2-log lower than S. pneumoniae $(1.26 \times$ $10^{3} \pm 10^{2}$ c.f.u. $\left./ \mathrm{g}\right)$, and it did not differ among the sampled lobes $(P=0.086)$. The associated median pulmonary histological score was 4 (interquartile range $4-4)(n=50$ samples). Thus, pneumococcal pneumonia was microbiologically/histologically confirmed in 8 of 10 infected pigs (80\%). In the remaining two pigs, pathology studies confirmed confluent pneumonia (i.e., a score $\geq 3$ ); however, the $S$. pneumoniae concentration was below the microbiological threshold (i.e., $<10^{3}$ c.f.u./g) to ultimately confirm pneumonia. Pneumococcal pneumonia was always multi-lobar. In control pigs, $S$. suis and $S$. aureus were the most commonly encountered pathogens. The mean \pm s.e.m. pulmonary concentration of pathogens in control pigs was $7.94 \pm 20$ c.f.u./g and slightly differed between the sampled lobes $(P=0.05)$.

Clinical findings, lung function and hemodynamics. Clinical and hemodynamic data are reported in Tables 1 and 2. Pneumonia development was not associated with specific changes in body temperature or white blood cells. Conversely, a significant drop in platelet count was observed in infected pigs. A significant increase in prothrombin time was another sign of systemic infection. In addition, creatinine was higher in control than in infected pigs at baseline, albeit without clinical relevance. Because of a significant decrease in systemic vascular resistance in infected pigs, mean \pm s.e.m. arterial pressure decreased to $66.9 \pm 6.8 \mathrm{mmHg}$ at $24 \mathrm{~h}$, whereas cardiac output significantly increased. In addition, mean pulmonary arterial pressure was significantly different between infected and control pigs. Norepinephrine was administered in 9 of 10 challenged pigs at some point during the experiment to sustain hemodynamic stability, whereas control pigs did not need vasopressor support during the entire experiment $(P=0.003)$. Venous saturation was kept within the normal range throughout the study in both infected and control groups. Finally, gas exchanges worsened in infected pigs, specifically at $24 \mathrm{~h}$ after bacterial challenge (Supplementary Fig. 2). A progressive and significant increase in ventilatory support was therefore necessary to sustain pulmonary function in those pigs (Supplementary Table 1).

Inflammatory biomarkers. Systemic inflammatory biomarkers significantly increased at $24 \mathrm{~h}$ in infected pigs compared with baseline due to $S$. pneumoniae challenge but decreased thereafter (Supplementary Fig. 3). There was a steady increase in tumor necrosis factor $\alpha$ throughout the study in the serum of infected pigs. In BAL fluids of infected pigs, IL- $1 \beta$, IL- 6 and IL- 8 concentrations significantly changed over time, reaching peak levels after 


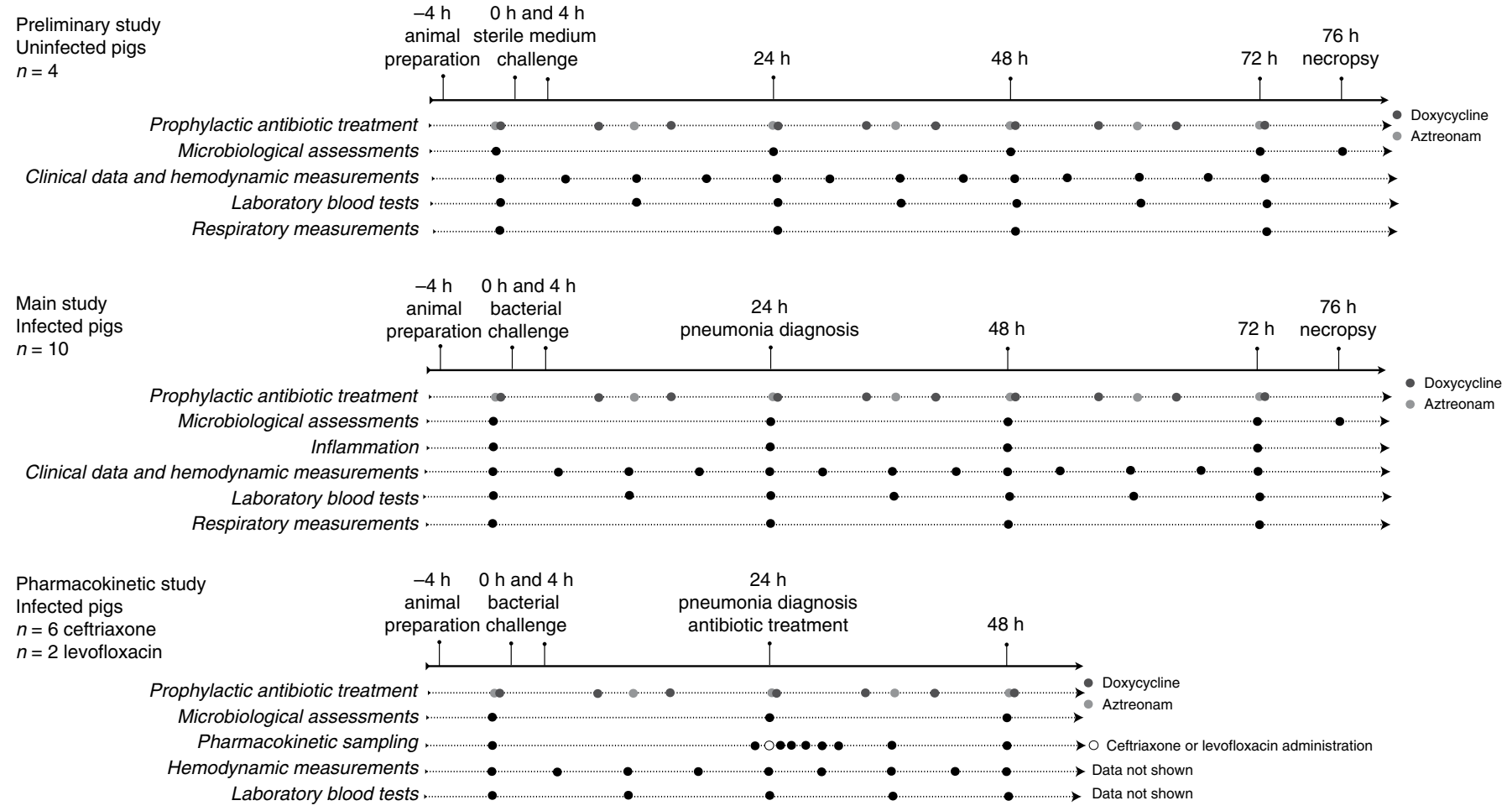

Fig. 1 | Study design of preliminary, main and pharmacokinetic studies. The timeline of experimental events is displayed. The number of pigs used for each study is reported. The preliminary and main studies were designed to compare data from microbiological assessments and other measurements between control pigs and pigs infected with S. pneumoniae serotype $19 \mathrm{~A}$. Of note, ceftriaxone pharmacokinetic studies were planned to achieve an epithelial lining fluid concentration above S. pneumoniae minimum inhibitory concentration for $\geq 60 \%$ of the time within $24 \mathrm{~h}$ in at least two consecutive pigs. For levofloxacin, we aimed for a ratio of area under the curve to minimum inhibitory concentration of bound levofloxacin $>40$.

$24 \mathrm{~h}$ of bacterial challenge and returning to baseline levels at $72 \mathrm{~h}$ (Supplementary Fig. 4).

Pharmacokinetic studies. A study of pharmacokinetics of ceftriaxone and levofloxacin was also performed (Fig. 6 and Supplementary Table 2). Six pigs were necessary to fulfill the expected goals of maintaining an unbound ceftriaxone epithelial lining fluid (ELF) concentration above $S$. pneumoniae minimum inhibitory concentration (MIC) for $\geq 60 \%$ of the time within $24 \mathrm{~h}$. Increasing doses of ceftriaxone-50, 100 and $150 \mathrm{mg} / \mathrm{kg}$-were therefore tested (i.e., two pigs per dose). Conversely, for levofloxacin, the first tested dose of $10 \mathrm{mg} / \mathrm{kg}$ ( $n=2$ pigs) was sufficient to achieve a ratio of area under the curve to MIC of bound levofloxacin $>40$.

\section{Discussion}

We developed a novel model of macrolide-resistant, multi-lobar pneumococcal pneumonia in mechanically ventilated pigs. Characterization of the model includes $S$. pneumoniae bacteremia in $50 \%$ of pigs and an important systemic and pulmonary release of inflammatory markers that causes septic shock and pulmonary/ hemodynamic derangement. The survival rate at $76 \mathrm{~h}$ was $70 \%$ in infected pigs, allowing for a relatively prolonged follow-up after the development of infection.

To the best of our knowledge, this is the first model of penicillinand macrolide-resistant pneumococcal pneumonia in animals ventilated for days, with severe hemodynamic impairment and requiring the use of vasoactive drugs. In the late 1970s, the first ground-breaking model of pneumococcal pneumonia was developed in anesthetized rats, via instillation of $S$. pneumoniae serotype 3 into the left main bronchus ${ }^{10}$. The animals were spontaneously breathing, and infection even spread into the right lung throughout the study, which lasted up to $132 \mathrm{~h}$. A detailed description of pneumococcal pneumonia in mice, rats and rabbits goes beyond the scope of this article, given previous comprehensive appraisals of animal models of $S$. pneumoniae infections ${ }^{10}$. Nevertheless, to determine the limitations of our novel model and identify possible refinements, taking a closer look at previously developed models of multi-lobar pneumococcal pneumonia that cause significant hemodynamic compromise is essential. The mouse models presenting with the most severe pulmonary infections were developed through intra-tracheal instillation of S. pneumoniae. Azoulay-Dupuis and colleagues $^{13}$ developed a model of severe pneumococcal pneumonia in Swiss mice via tracheal cannulation and instillation of $S$. pneumoniae type 3 with microliter syringes. In another model, Iwasaki and collaborators ${ }^{14}$ lightly sedated a mouse and introduced an otoscope into the mouse's oropharynx for direct visualization of the trachea and bacterial challenge into the airways.

In comparison with our model, though, several dissimilarities are evident. First, neither mice nor rats underwent mechanical ventilation, and circulatory shock was not supported by administering vasoactive drugs. Second, severe pneumonia developed in just 8-12 h in our swine model, whereas mice and rats required days to develop lethal severity. Furthermore, our model used a clinically relevant, penicillin- and macrolide-resistant $S$. pneumoniae serotype (19 A). There are well-known difficulties in causing pneumonia in rodents with penicillin-resistant $S$. pneumoniae, and scientists have ultimately used immunocompromised or young rodents to develop infections with such a strain ${ }^{10}$.

Alternatively, rabbitscan be used to study pneumoniaby penicillinresistant $S$. pneumoniae in immune-competent animals ${ }^{15,16}$. For example, Piroth and collaborators ${ }^{16}$ undertook an extremely high intrabronchial challenge approach of $10^{10}$ c.f.u./ml of penicillinresistant $S$. pneumoniae serotype $9 \mathrm{~V}$. After $72 \mathrm{~h}$, the authors reported a survival rate of $50 \%$ and progression from lobar to multi-lobar 

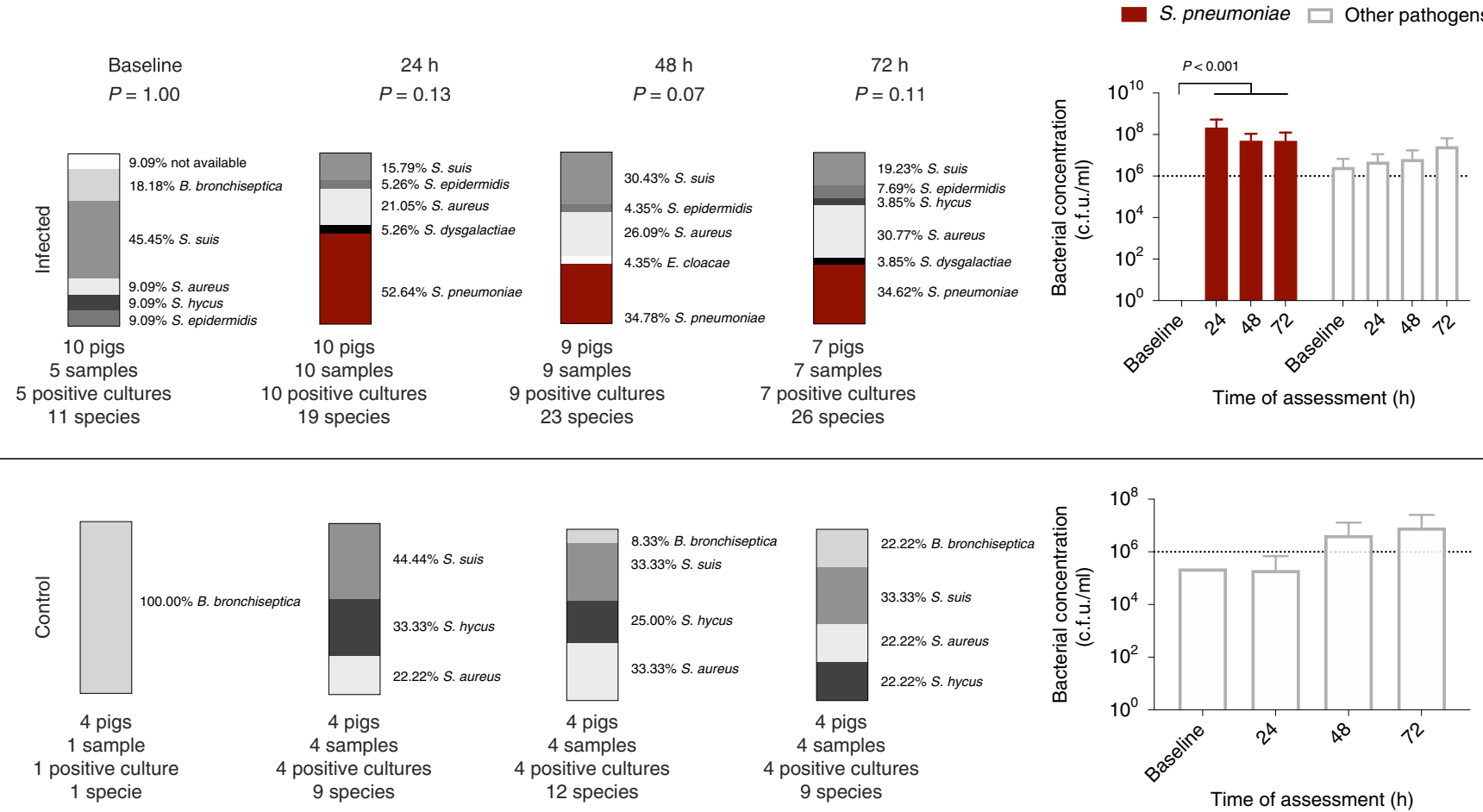

Fig. 2 | Bacterial colonization of tracheal secretions. On the upper left side, tracheal secretion bacterial diversity during the main study is shown for S. pneumoniae-infected pigs. On the lower left side, tracheal secretion bacterial diversity during the preliminary study is shown for control pigs. In the right section, sequential assessment of quantitative bacterial concentration in tracheal secretions in infected (upper plot) and control (lower plot) pigs is shown (mean \pm s.e.m. bars). In infected pigs, the concentration of S. pneumoniae (red bars) in tracheal secretion increased after bacterial challenge $(P<0.001)$, whereas the concentrations of other pathogens (gray bars) did not vary between study time points $(P=0.53)$. In controls, bacterial concentration did not vary during study times $(P=0.59)$. Data are reported as percentage of isolated bacteria among total tracheal aspirate samples as a pool of pigs combined. The number of included pigs, analyzed samples, positive cultures and isolated species are indicated for each group and time of assessment. The percentage of colonization between infected and control group variables was analyzed by using Fisher's exact test and the CochranMantel-Haenszel test. Restricted maximum likelihood (REML) analysis based on a repeated measures approach with time of assessment as factors was performed. $P$ values - corrected through Bonferroni methods-for comparisons between bacterial burden per study time are reported. B. bronchiseptica, Bordetella bronchiseptica; E. cloacae, Enterobacter cloacae; S. aureus, Staphylococcus aureus; S. dysgalactiae, Streptococcus dysgalactiae; S. epidermidis, Staphylococcus epidermidis; S. hycus, Staphylococcus hycus; S. pneumoniae, Streptococcus pneumoniae; S. suis, Streptococcus suis.

pneumonia. Yet, even then, rabbits did not receive mechanical ventilation or care that would be provided to patients in ICU.

With respect to models of pneumococcal pneumonia in larger animals, Kraft et al. developed a pneumococcal pneumonia model in adult baboons (Papio cynocephalus) that were infected with escalating doses of $S$. pneumoniae serotype $19 \mathrm{~A}^{17}$. Although this model is characterized by a good association between dose inoculation and cytokine response, the baboons were not mechanically ventilated and were diagnosed by using traditional methods, and no microbiological data of lung burden are available. Similarly, Reyes and collaborators ${ }^{18}$ described an innovative model in adult baboons that underwent bacterial challenge with $10^{9}$ c.f.u. $/ \mathrm{ml}$ of $S$. pneumoniae serotype 4 in the right middle lobe. The baboons developed severe pneumonia and bacteremia but not septic shock; no mechanical ventilation was necessary. Furthermore, it is uncertain whether these nonhuman primates developed concomitant colonization by endogenous pathogens. Lim et al. ${ }^{19}$ also developed an S. pneumoniae model in pigs by challenging each pulmonary lower lobe with $10^{8}$ c.f.u. $/ \mathrm{ml}$. Unlike the previous studies, these experiments lasted only $6 \mathrm{~h}$, and no microbiological information was reported.

In our study, we aimed at creating a highly severe model of pneumococcal pneumonia, similar to that observed in patients requiring intensive care. Indeed, between $1.2 \%$ and $10.0 \%$ of patients requiring hospital admission for CAP caused by $S$. pneumoniae will require ICU admission, and 28-d mortality in patients admitted to the ICU is $17 \%$. This figure increases to $25 \%$ in patients who require invasive mechanical ventilation and $40 \%$ in patients who develop septic shock $^{20}$. The ideal treatment for severe pneumococcal pneumonia is limited to antibiotic therapy and potential ventilatory support until pulmonary function has recovered. Furthermore, current therapeutic options for severe pneumococcal pneumonia still lack an evidence-based guideline for best practice. None of the previously described models underwent mechanical ventilation for $>6$ h. Therefore, our reliable model of pneumococcal pneumonia may serve as a means to obtain experimental results that would be likely unachievable in clinical settings, particularly because this animal model can be used to harvest various tissues post mortem ${ }^{21}$.

We used prophylactic antibiotic therapy with doxycycline and aztreonam to avoid colonization by species-specific endogenous pathogens. As clearly demonstrated by our results obtained from control pigs, these antibiotics adequately prevented infection, and all pigs survived the 76-h experiment. Nevertheless, in pigs with pneumococcal pneumonia, the concentration of endogenous flora increased, although always marginally when compared to $S$. pneumoniae. Prophylactic antibiotics were not effective enough to prevent infection of an already injured lung by $S$. pneumoniae. Endogenous flora probably occurs in mechanically ventilated patients, as suggested by post-mortem studies ${ }^{22}$. The interplay between endogenous flora and $S$. pneumoniae cannot be fully described with our current study design. Lung damage caused by 

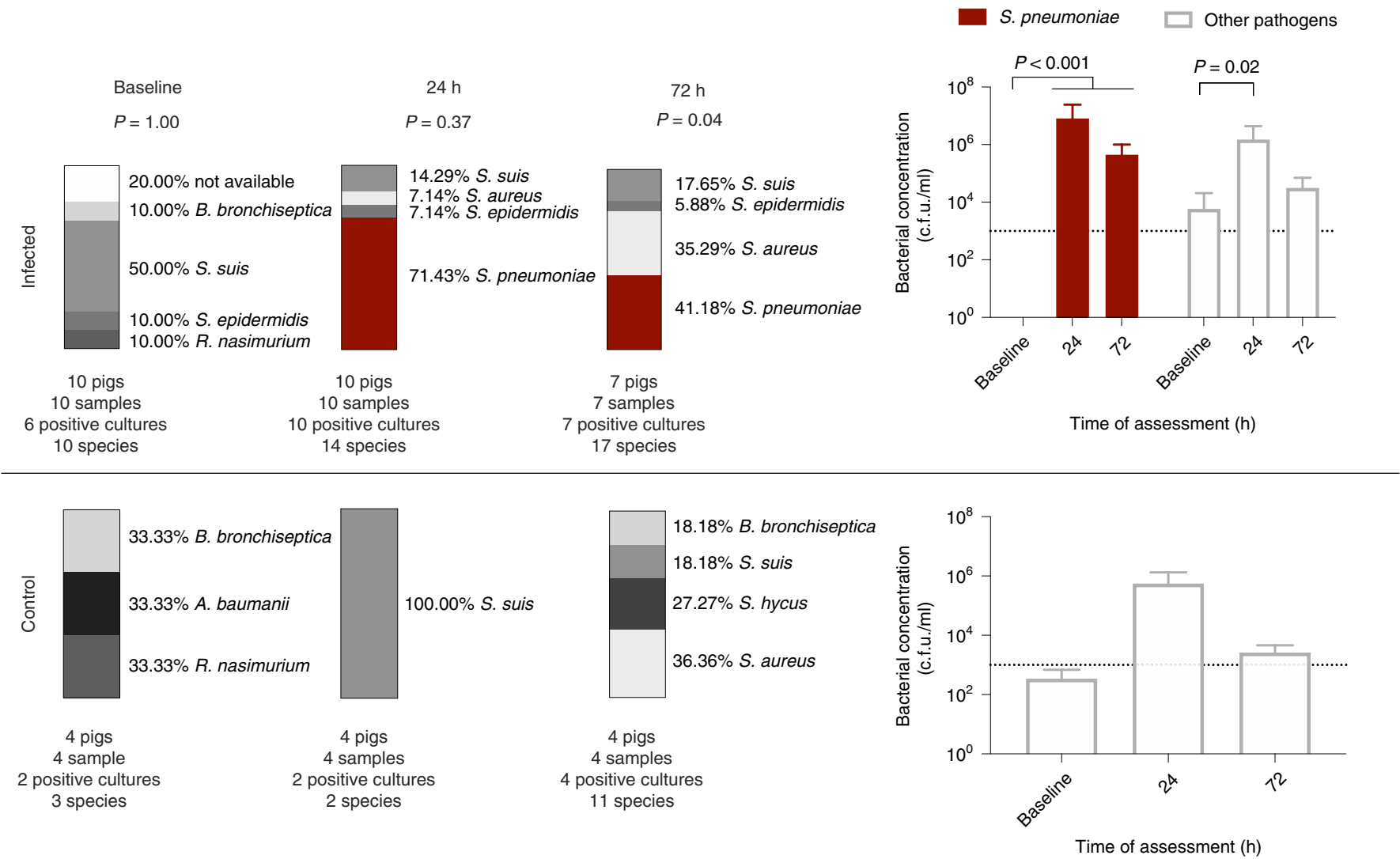

Fig. 3 | Bacterial colonization of BAL. The upper left section depicts BAL bacterial diversity during the main study in infected pigs. On the lower left side, BAL bacterial diversity during the preliminary study is shown for control pigs. In the right section, sequential assessment of quantitative bacterial concentration in BAL fluids in infected (upper plot) and control (lower plot) pigs is shown (mean \pm s.e.m. bars). In infected pigs, the concentration of S. pneumoniae (red bars) in BAL fluids increased after bacterial challenge $(P<0.001)$, whereas for other pathogen concentrations (gray bars), the burden increased progressively between study times $(P=0.02)$, specifically between baseline and $24 \mathrm{~h}(P=0.02)$. In controls, bacterial concentration did not vary during study times $(P=0.08)$. Data are reported as percentage of isolated bacteria among total $B A L$ samples as a pool of pigs combined. The number of included pigs, analyzed samples, positive cultures and isolated species are indicated for each group and time of assessment. The percentage of colonization between infected and control group variables was analyzed by using Fisher's exact test and the Cochran-Mantel-Haenszel test. REML analysis based on a repeated measures approach with time of assessment as factors was performed. $P$ values-corrected through Bonferroni methodsfor comparisons between bacterial burden per study time are reported. A. baumanii, Acinetobacter baumannii; R. nasimurium, Rothia nasimurium.

S. pneumoniae may facilitate the colonization of the lower airway by endogenous flora. In this context, studies have shown that patients with greater lung damage (i.e., patients with acute respiratory distress syndrome) have a higher risk of infection by more than one pathogen than patients with lower pulmonary insult ${ }^{23}$. Significant differences between baseline and positive BAL cultures $24 \mathrm{~h}$ after bacterial challenge corroborate this hypothesis. Therefore, it is possible that the resulting, life-threatening pulmonary insult performed by two consecutive bacterial challenges significantly thwarted respiratory defenses and facilitated endogenous colonization in our model. Future studies should therefore consider the underlying pathophysiological mechanisms for concurrent bacterial growth. Furthermore, in view of the high $S$. aureus colonization in infected pigs, our model may set the groundwork to test breakthrough fifth generation antibiotics active against both pathogens (e.g., ceftobiprole ${ }^{24}$ or ceftaroline ${ }^{25}$ ) and potentially detect their effect on both S. pneumoniae and S. aureus burden.

An additional strength of our model is the use of an $S$. pneumoniae strain resistant to penicillin and macrolides. Our model contributes to providing insight into macrolide resistance in CAP. Previous investigations have reported macrolide-resistant S. pneumoniae rates to be between $15 \%$ and $35 \%{ }^{26}$. These figures are alarming, given that macrolides are first-line antibiotic treatment in patients with $\mathrm{CAP}^{27}$. In addition, results related to the effects of macrolide resistance on patient outcomes have been conflicting. Our model, however, could help explore these controversial matters or even address potential immunomodulatory benefits associated with macrolides ${ }^{28}$ while even preventing the bactericidal effects of such drugs. Finally, as in previous models ${ }^{29}$, our model could allow investigators to easily study the deleterious cardiac effects and potential remodeling caused by severe pneumococcal pneumonia.

As for other noteworthy clinical features of our model, we observed hyperdynamic septic shock at $24 \mathrm{~h}$, characterized by a fall in mean arterial pressure (MAP) and systemic vascular resistance and an increase in cardiac output. This is in line with the clinical status of patients admitted to the ICU with pneumococcal pneumonia, who often require the use of vasopressors. We also found sepsis-induced coagulopathy, as corroborated by prolonged prothrombin time and thrombocytopenia. However, possibly because of prompt hemodynamic support during the development of infection, no other clinical signs of organ failure were evident. In addition, we confirmed systemic inflammatory response on the basis of a considerable upsurge of inflammatory markers IL-1ß, IL-6 and IL-8. This model could therefore be useful in the testing of novel strategies aimed at modulating inflammation during the early disease stages. Moreover, novel antimicrobial agents with 

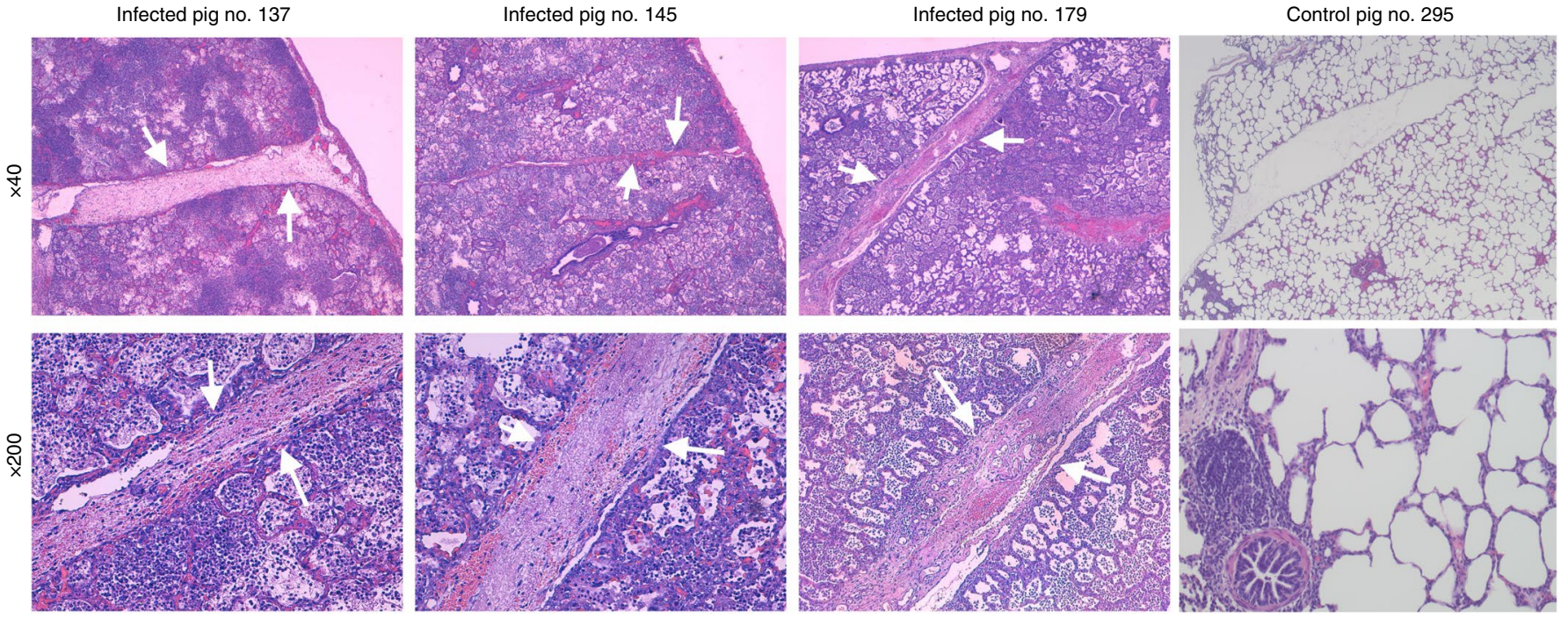

Fig. 4 | Lung tissue microscopic findings from infected pigs (nos. 137, 145 and 179) and a control pig (no. 295). Upper figures show $\times 40$ magnification, and lower figures show $\times 200$ magnification. Confluent pneumonia was the most common histological pattern in infected pigs: polymorphonuclear infiltrate is identifiable within contiguous secondary lobes, separated by a septum (white arrows indicate the septum margins). Findings in control pigs included mild bronchiolitis, composed of histocytes with some polymorphs, and interlobular septal edema.

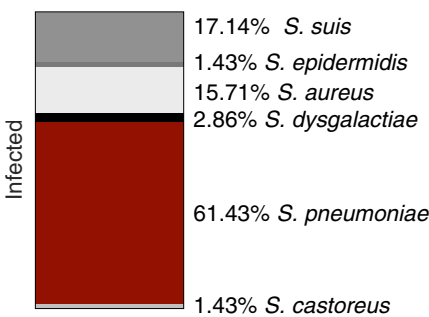

10 pigs 50 lobes 46 positive cultures 70 species

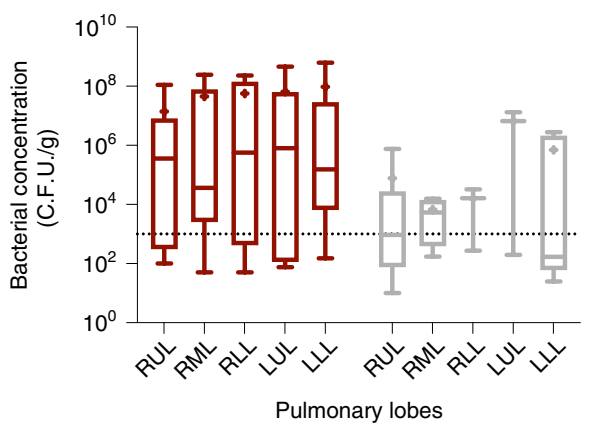

Pulmonary lobes
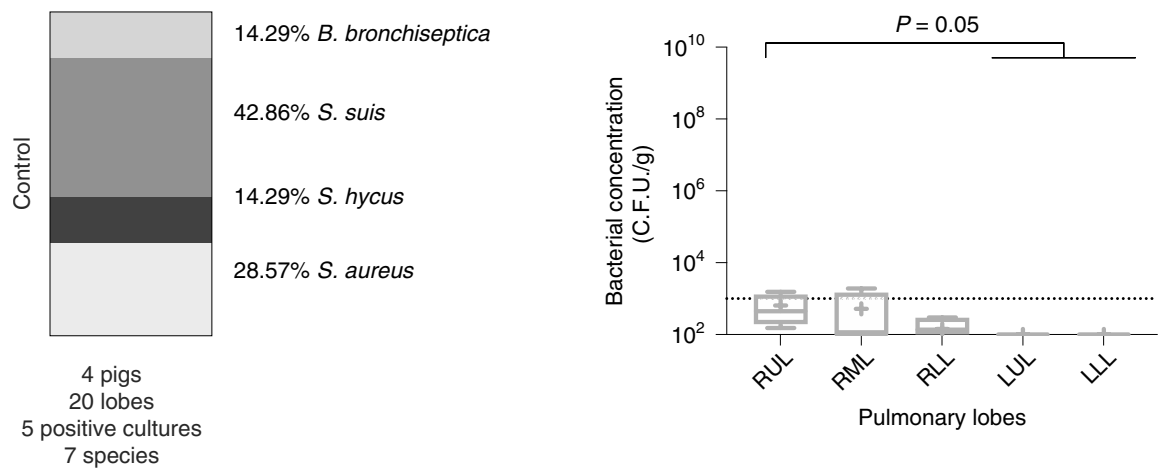

Fig. 5 | Pulmonary microbiological burden. The left section depicts pulmonary bacterial variety. In the right section, box plots display pulmonary concentration per lobes. Horizontal bars represent the median, boxes represent the interquartile range, whiskers represent the range and plus signs denote the means. In the upper graph, red box plots depict lobar S. pneumoniae concentration data, whereas gray ones depict other concomitant pathogens per lobes among infected pigs. Neither pulmonary S. pneumoniae concentration $(P=0.92)$ nor concomitant pathogen concentrations $(P=0.09)$ differed between pulmonary lobes. In the lower right section, the pulmonary concentration of other pathogens is shown for the control group. Significant differences were found between the right upper lobe (RUL) and the left upper lobe (LUL) and left lower lobe (LLL) $(P=0.05)$. Data are reported as percentage of isolated bacteria among lung tissue samples as a pool of pigs combined. The number of included pigs, analyzed pulmonary lobes, positive cultures and isolated species are indicated for each group and time of assessment. REML analysis based on a repeated measures approach with lung lobes as factors was performed. $P$ values-corrected through Bonferroni methods-for comparisons between bacterial burden per lung lobes are reported. S. castoreus, Streptococcus castoreus. RML, right middle lobe; RLL, right lower lobe. 
Table 1 | Clinical data

\begin{tabular}{|c|c|c|c|c|c|c|c|c|c|c|}
\hline & \multicolumn{8}{|l|}{ Time (h) } & \multirow{2}{*}{\multicolumn{2}{|c|}{$P$ value }} \\
\hline & \multicolumn{2}{|l|}{0} & \multicolumn{2}{|l|}{24} & \multicolumn{2}{|l|}{48} & \multicolumn{2}{|l|}{72} & & \\
\hline & $\begin{array}{l}\text { Infected } \\
(n=10)\end{array}$ & $\begin{array}{l}\text { Control } \\
(n=4)\end{array}$ & $\begin{array}{l}\text { Infected } \\
(n=10)\end{array}$ & $\begin{array}{l}\text { Control } \\
(n=4)\end{array}$ & $\begin{array}{l}\text { Infected } \\
(n=9)\end{array}$ & $\begin{array}{l}\text { Control } \\
(n=4)\end{array}$ & $\begin{array}{l}\text { Infected } \\
(n=7)\end{array}$ & $\begin{array}{l}\text { Control } \\
(n=4)\end{array}$ & $\begin{array}{l}\text { Effect of } \\
\text { bacterial } \\
\text { challenge }\end{array}$ & $\begin{array}{l}\text { Effect of } \\
\text { time }\end{array}$ \\
\hline $\begin{array}{l}\text { Body } \\
\text { temperature }\left({ }^{\circ} \mathrm{C}\right)\end{array}$ & $36.8 \pm 0.9$ & $37.4 \pm 0.6$ & $38.7 \pm 0.6$ & $39.1 \pm 0.9$ & $38.6 \pm 0.7$ & $38.7 \pm 0.5$ & $38.6 \pm 0.5$ & $37.1 \pm 1.4$ & 0.693 & $<0.001$ \\
\hline $\begin{array}{l}\text { Leukocytes } \\
\left(10^{3} / \mu \mathrm{l}\right)\end{array}$ & $10.9 \pm 4.6$ & $9.1 \pm 1.2$ & $8.3 \pm 2.3$ & $23.0 \pm 3.3$ & $19.2 \pm 8.7$ & $13.3 \pm 4.6$ & $13.3 \pm 6.1$ & $10.2 \pm 3.2$ & 0.542 & 0.002 \\
\hline $\begin{array}{l}\text { Creatinine } \\
(\mathrm{mg} / \mathrm{dl})\end{array}$ & $0.8 \pm 0.1$ & $1.1 \pm 0.1$ & $0.9 \pm 0.1$ & $1.2 \pm 0.3$ & $0.9 \pm 0.1$ & $1.1 \pm 0.3$ & $0.9 \pm 0.1$ & $1.1 \pm 0.4$ & $<0.001$ & 0.185 \\
\hline ALT (IU/liter) & $31.3 \pm 9.4$ & $34.0 \pm 16.5$ & $28.8 \pm 5.8$ & $29.0 \pm 21.2$ & $34.4 \pm 10.9$ & $26.7 \pm 13.0$ & $36.3 \pm 15.4$ & $51.0 \pm 39.1$ & 0.654 & 0.209 \\
\hline $\begin{array}{l}\text { Total bilirubin } \\
(\mathrm{mg} / \mathrm{dl})\end{array}$ & $0.13 \pm 1.03$ & $0.20 \pm 0.10$ & $0.21 \pm 0.14$ & $0.10 \pm 0.0$ & $0.11 \pm 0.66$ & $0.12 \pm 0.05$ & $0.12 \pm 0.04$ & $0.12 \pm 0.05$ & 0.304 & 0.749 \\
\hline
\end{tabular}

Data are reported as mean \pm s.d. Two infected pigs were euthanized at 34 and $48 \mathrm{~h}$ for severe respiratory/hemodynamic instability, and one infected pig was euthanized at $55 \mathrm{~h}$ because of severe hemodynamic instability. Restricted maximum likelihood analysis based on a repeated measures approach with time of assessment as factors was performed. Pair-wise comparisons corrected by Bonferroni were performed for both bacterial challenge and time. Pair-wise comparisons corrected by Bonferroni between infected and control pigs among study time points are displayed in bold. ALT, alanine transaminase; PT, prothrombin time; PTT, thromboplastin time.

Table 2 | Hemodynamic parameters

\begin{tabular}{|c|c|c|c|c|c|c|c|c|c|c|}
\hline & \multicolumn{8}{|l|}{ Time (h) } & \multirow{2}{*}{\multicolumn{2}{|c|}{$P$ value }} \\
\hline & \multicolumn{2}{|l|}{0} & \multicolumn{2}{|l|}{24} & \multicolumn{2}{|l|}{48} & \multicolumn{2}{|l|}{72} & & \\
\hline & $\begin{array}{l}\text { Infected } \\
(n=10)\end{array}$ & $\begin{array}{l}\text { Control } \\
(n=4)\end{array}$ & $\begin{array}{l}\text { Infected } \\
(n=10)\end{array}$ & $\begin{array}{l}\text { Control } \\
(n=4)\end{array}$ & $\begin{array}{l}\text { Infected } \\
(n=9)\end{array}$ & $\begin{array}{l}\text { Control } \\
(n=4)\end{array}$ & $\begin{array}{l}\text { Infected } \\
(n=7)\end{array}$ & $\begin{array}{l}\text { Control } \\
(n=4)\end{array}$ & $\begin{array}{l}\text { Effect of } \\
\text { bacterial } \\
\text { challenge }\end{array}$ & $\begin{array}{l}\text { Effect of } \\
\text { time }\end{array}$ \\
\hline HR (beats/min) & $56.3 \pm 11.9$ & $75.2 \pm 20.6$ & $90.7 \pm 20.3$ & $77.2 \pm 28.6$ & $69.2 \pm 13.5$ & $52.7 \pm 10.8$ & $60.3 \pm 9.5$ & $49.2 \pm 7.5$ & 0.264 & $<0.001$ \\
\hline $\mathrm{MAP}(\mathrm{mmHg})$ & $82.4 \pm 10.7$ & $83.7 \pm 1.5$ & $66.9 \pm 6.8$ & $69.5 \pm 1.2$ & $67.9 \pm 4.7$ & $68.2 \pm 1.7$ & $71.0 \pm 6.7$ & $77.2 \pm 3.8$ & 0.233 & $<0.001$ \\
\hline $\operatorname{MPAP}(\mathrm{mmHg})$ & $15.9 \pm 5.1$ & $13.6 \pm 4.1$ & $24.8 \pm 3.2$ & $19.4 \pm 5.6$ & $23.7 \pm 3.9$ & $17.5 \pm 3.1$ & $23.0 \pm 2.54$ & $18.4 \pm 3.1$ & $<0.001$ & $<0.001$ \\
\hline CO (liters/min) & $3.6 \pm 1.5$ & $3.1 \pm 0.8$ & $4.8 \pm 1.7$ & $2.4 \pm 0.6$ & $4.6 \pm 2.0$ & $2.6 \pm 0.6$ & $3.8 \pm 1.4$ & $2.3 \pm 0.3$ & 0.001 & 0.769 \\
\hline SVR (dyn's.cm-5) & $1883 \pm 607$ & $2249 \pm 583$ & $1112 \pm 461$ & $2075 \pm 394$ & $1146 \pm 364$ & $1842 \pm 239$ & $1436 \pm 433$ & $2363 \pm 381$ & $<0.001$ & 0.002 \\
\hline PVR (dyn's.cm-5) & $217 \pm 112$ & $259 \pm 22$ & $261 \pm 87$ & $303 \pm 48$ & $239 \pm 86$ & $193 \pm 31$ & $272 \pm 84$ & $279 \pm 34$ & 0.652 & 0.206 \\
\hline $\begin{array}{l}\text { Mixed venous } \\
\text { SatO2 (\%) }\end{array}$ & $66.9 \pm 3.3$ & $72.0 \pm 8.2$ & $74.6 \pm 9.2$ & $67.4 \pm 5.3$ & $66.2 \pm 9.3$ & $65.6 \pm 10.2$ & $68.4 \pm 4.4$ & $70.1 \pm 6.3$ & 0.913 & 0.478 \\
\hline
\end{tabular}

Data are reported as mean \pm s.d. or median and (interquartile range). Two infected pigs were euthanized at 34 and $48 \mathrm{~h}$ for severe respiratory/hemodynamic instability, and one infected pig was euthanized at $55 \mathrm{~h}$ because of severe hemodynamic instability. REML analysis based on a repeated measures approach with time of assessment as factors was performed. Pair-wise comparisons corrected by Bonferroni between infected and control pigs among study time points are displayed in bold. CO, cardiac output; HR, heart rate; MAP, mean arterial pressure; MPAP, mean pulmonary arterial pressure; $\mathrm{PVR}$, pulmonary vascular resistance; $\mathrm{SatO}_{2}$, oxygen saturation; $\mathrm{SVR}$, systemic vascular resistance.

Gram-positive or broader-spectrum features (e.g., ceftaroline and ceftobiprole or omadacycline, respectively) could be tested in future studies.

Finally, another major strength of our study is the assessment of ELF concentrations of ceftriaxone and levofloxacin. These analyses serve as proof of concept for the potential use of this novel model. In particular, as detailed in Methods, we aimed for specific endpoints commonly applied in clinical settings to ensure bactericidal efficacy, as is normally done in the first phases of clinical trials. Interestingly, in comparison with dosages in humans, a fourfold increase in ceftriaxone dose was necessary to achieve a concentration of free antibiotic above S. pneumoniae MIC for $60 \%$ of the time. This was probably related to the substantial severity and resulting hyperdynamic septic shock of our model ${ }^{30}$, largely augmenting the distribution volume of ceftriaxone. These data also highlight the potential risks of under-dosing in critically ill patients and the need for new reliable clinical data to validate ELF concentrations. In addition, these findings are in line with previously reported lower pulmonary concentrations and efficacy attained with standard daily dosing of $2 \mathrm{~g}$ of ceftriaxone in the treatment of $S$. aureus pneumonia ${ }^{31,32}$. Conversely, required levofloxacin doses were similar to doses used in patients.

This study presents some limitations that deserve further discussion. First, unlike in the most probable clinical scenario, we used a high challenge dose in ventilated pigs, which resulted in an immediate injurious response. These methods were aimed at developing severe pneumococcal pneumonia quickly, and the model should be considered to have captured only certain features of pneumococcal 

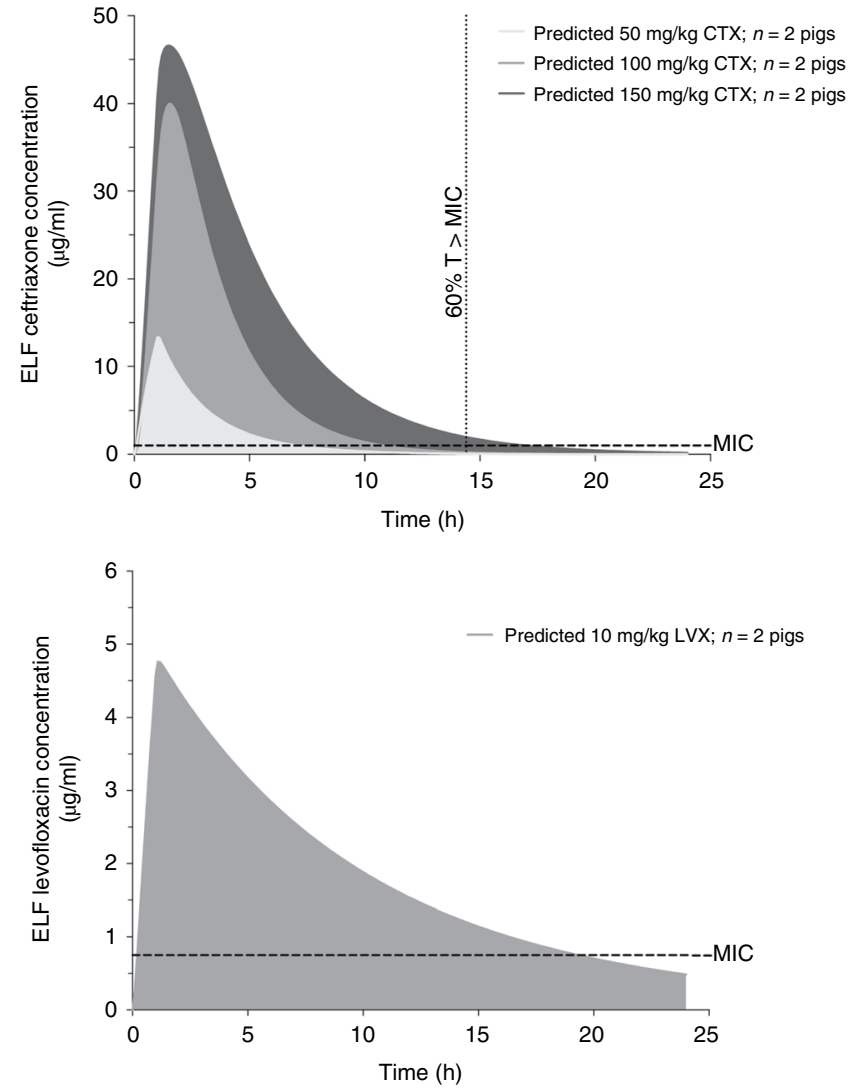

Fig. 6 | Epithelial lining fluid (ELF) pharmacokinetics of ceftriaxone and levofloxacin. In the upper portion of the figure, ceftriaxone (CTX) pharmacokinetics are shown. A dose of $150 \mathrm{mg} / \mathrm{kg}$ of ceftriaxone was necessary to achieve a concentration of unbound ceftriaxone concentration above S. pneumoniae minimum inhibitory concentration (MIC) for $\geq 60 \%$ of the time between daily dosing ( $y$-axis dotted line, ' $60 \% \mathrm{~T}>M I C^{\prime}$ ). Levofloxacin (LVX) pharmacokinetics are shown in the lower portion of the figure. A dose of $10 \mathrm{mg} / \mathrm{kg}$ was sufficient to achieve the ratio between the area under the curve for $0-24 \mathrm{~h}$ and S. pneumoniae MIC of $\geq 40$ (y-axis dotted line).

pneumonia. Second, we found that $S$. aureus and S. suis concurrently colonized the respiratory system. An alternative methodological approach could consist of further increasing the doxycycline dose to avoid colonization by the most frequent Gram-positive pathogens. Third, because of severe respiratory/hemodynamic instability, we found a survival rate of $70 \%$. This substantial mortality may be a limitation for future drug-testing studies, by reducing the number of animals included in the analysis, raising ethical concerns and creating potential bias in results. Fourth, only female pigs were used, and thus the impact of sex on the model is unknown. Finally, potential viral colonization or coinfection has not been studied.

In conclusion, we have developed a model of penicillin- and macrolide-resistant S. pneumoniae serotype 19 A in mechanically ventilated pigs. Pulmonary infection caused bacteremia in half of the pigs and was associated with severe hemodynamic compromise.

\section{Online content}

Any methods, additional references, Nature Research reporting summaries, source data, extended data, supplementary information, acknowledgements, peer review information; details of author contributions and competing interests; and statements of data and code availability are available at https://doi.org/10.1038/ s41684-021-00876-y.
Received: 16 September 2020; Accepted: 16 September 2021; Published online: 21 October 2021

\section{References}

1. Cilloniz, C. et al. Microbial aetiology of community-acquired pneumonia and its relation to severity. Thorax 66, 340-346 (2011).

2. Feikin, D. R. et al. Mortality from invasive pneumococcal pneumonia in the era of antibiotic resistance, 1995-1997. Am. J. Public Health 90, 223-229 (2000).

3. Bedos, J. P. et al. Host-pathogen interactions and prognosis of critically ill immunocompetent patients with pneumococcal pneumonia: the nationwide prospective observational STREPTOGENE study. Intensive Care Med. 44, 2162-2173 (2018)

4. Schroeder, M. R. \& Stephens, D. S. Macrolide resistance in Streptococcus pneumoniae. Front. Cell. Infect. Microbiol. 6, 98 (2016).

5. Schroeder, M. R. et al. A population-based assessment of the impact of 7- and 13-valent pneumococcal conjugate vaccines on macrolide-resistant invasive pneumococcal disease: emergence and decline of Streptococcus pneumoniae serotype 19A (CC320) with dual macrolide resistance mechanisms. Clin. Infect. Dis. 65, 990-998 (2017).

6. Naucler, P. et al. Comparison of the impact of pneumococcal conjugate vaccine 10 or pneumococcal conjugate vaccine 13 on invasive pneumococcal disease in equivalent populations. Clin. Infect. Dis. 65, 1780-1789 (2017).

7. Aliberti, S., Mantero, M., Mirsaeidi, M. \& Blasi, F. The role of vaccination in preventing pneumococcal disease in adults. Clin. Microbiol. Infect. 20, 52-58 (2014).

8. Fenoll, A. et al. Temporal trends of invasive Streptococcus pneumoniae serotypes and antimicrobial resistance patterns in Spain from 1979 to 2007 J. Clin. Microbiol. 47, 1012-1020 (2009).

9. Quah, J., Jiang, B., Tan, P. C., Siau, C. \& Tan, T. Y. Impact of microbial Aetiology on mortality in severe community-acquired pneumonia. BMC Infect. Dis. 18, 451 (2018).

10. Chiavolini, D., Pozzi, G. \& Ricci, S. Animal models of Streptococcus pneumoniae disease. Clin. Microbiol. Rev. 21, 666-685 (2008).

11. Luna, C. M. et al. Experimental severe Pseudomonas aeruginosa pneumonia and antibiotic therapy in piglets receiving mechanical ventilation. Chest 132, 523-531 (2007).

12. Martinez-Olondris, P. et al. An experimental model of pneumonia induced by methicillin-resistant Staphylococcus aureus in ventilated piglets. Eur. Respir. J. 36, 901-906 (2010).

13. Azoulay-Dupuis, E., Bedos, J. P., Vallee, E. \& Pocidalo, J. J. Comparative activity of fluorinated quinolones in acute and subacute Streptococcus pneumoniae pneumonia models: efficacy of temafloxacin. J. Antimicrob. Chemother. 28, 45-53 (1991).

14. Iwasaki, K. et al. Combined effects of both bacteria and gastric juice on pneumonia in mice. Respir. Physiol. 116, 201-209 (1999).

15. Croisier, D. et al. Mutant selection window in levofloxacin and moxifloxacin treatments of experimental pneumococcal pneumonia in a rabbit model of human therapy. Antimicrob. Agents Chemother. 48, 1699-1707 (2004).

16. Piroth, L. et al. Development of a new experimental model of penicillin-resistant Streptococcus pneumoniae pneumonia and amoxicillin treatment by reproducing human pharmacokinetics. Antimicrob. Agents Chemother. 43, 2484-2492 (1999).

17. Kraft, B. D. et al. Development of a novel preclinical model of pneumococcal pneumonia in nonhuman primates. Am. J. Respir. Cell Mol. Biol. 50, 995-1004 (2014).

18. Reyes, L. F. et al. A non-human primate model of severe pneumococcal pneumonia. PLoS One 11, e0166092 (2016).

19. Lim, S. C. et al. Transient hemodynamic effects of recruitment maneuvers in three experimental models of acute lung injury. Crit. Care Med. 32, 2378-2384 (2004).

20. Walden, A. P. et al. Patients with community acquired pneumonia admitted to European intensive care units: an epidemiological survey of the GenOSept cohort. Crit. Care 18, R58 (2014).

21. Yang, M. et al. Corticosteroid therapy combined with antibiotics for severe Streptococcus pneumoniae pneumonia in ventilated piglets. Eur. Respir. J. 54, PA4553 (2019).

22. Torres, A. et al. Sampling methods for ventilator-associated pneumonia: validation using different histologic and microbiological references. Crit. Care Med. 28, 2799-2804 (2000).

23. Cilloniz, C. et al. Community-acquired polymicrobial pneumonia in the intensive care unit: aetiology and prognosis. Crit. Care 15, R209 (2011).

24. Nicholson, S. C. et al. A randomised, double-blind trial comparing ceftobiprole medocaril with ceftriaxone with or without linezolid for the treatment of patients with community-acquired pneumonia requiring hospitalisation. Int. J. Antimicrob. Agents 39, 240-246 (2012). 
25. MacGowan, A. P., Noel, A. R., Tomaselli, S. \& Bowker, K. E. Pharmacodynamics of ceftaroline against Staphylococcus aureus studied in an in vitro pharmacokinetic model of infection. Antimicrob. Agents Chemother. 57, 2451-2456 (2013).

26. Cilloniz, C. et al. The effect of macrolide resistance on the presentation and outcome of patients hospitalized for Streptococcus pneumoniae pneumonia. Am. J. Respir. Crit. Care Med. 191, 1265-1272 (2015).

27. Mandell, L. A. et al. Infectious Diseases Society of America/American Thoracic Society consensus guidelines on the management of communityacquired pneumonia in adults. Clin. Infect. Dis. 44, S27-S72 (2007).

28. Zimmermann, P., Ziesenitz, V. C., Curtis, N. \& Ritz, N. The immunomodulatory effects of macrolides-a systematic review of the underlying mechanisms. Front. Immunol. 9, 302 (2018).

29. Reyes, L. F. et al. Severe pneumococcal pneumonia causes acute cardiac toxicity and subsequent cardiac remodeling. Am. J. Respir. Crit. Care Med. 196, 609-620 (2017).
30. Roberts, J. A. \& Roberts, D. M. Antibiotic dosing in critically ill patients with septic shock and on continuous renal replacement therapy: can we resolve this problem with pharmacokinetic studies and dosing guidelines? Crit. Care 18, 156 (2014).

31. So, W., Crandon, J. L. \& Nicolau, D. P. Poor outcomes of empiric ceftriaxone +/- azithromycin for community-acquired pneumonia caused by methicillin-susceptible Staphylococcus aureus. Intern. Emerg. Med. 11, 545-551 (2016)

32. Owens, R. C. Jr. et al. Pharmacodynamics of ceftriaxone and cefixime against community-acquired respiratory tract pathogens. Int. J. Antimicrob. Agents 17, 483-489 (2001).

Publisher's note Springer Nature remains neutral with regard to jurisdictional claims in published maps and institutional affiliations.

(c) The Author(s), under exclusive licence to Springer Nature America, Inc. 2021 


\section{Methods}

This study was conducted at the animal facilities of the University of Barcelona, Spain. All procedures were performed following European Directive 2010/63/ UE and Spanish RD 53/2013 regulations related to the Guide for the Care and Use of Laboratory Animals. The study protocol was approved by the Animal Experimentation Ethics Committee of the University of Barcelona (approval reference number: DAAM 42/12). To ensure adequate animal welfare, the pigs were housed in groups before the experiments. The pigs were tested for common pathogens before the experiments, identifying normal and non-pathogenic flora (data not shown). Figure 1 depicts the study design and aims of this multi-phase project. Further methodological details are available in the Supplementary Information

Preliminary study. We conducted preliminary analyses in four healthy Large White-Landrace female pigs (mean \pm s.e.m.: $31.5 \pm 3.0 \mathrm{~kg}$; range: $28-34 \mathrm{~kg}$; Specipig SL) that were surgically prepared and managed as reported below, but were not challenged intra-bronchially with $S$. pneumoniae. Throughout the study, $100 \mathrm{mg} / \mathrm{kg}$ of doxycycline was administered every $12 \mathrm{~h}$ to hinder colonization by endogenous Gram-positive bacteria. In addition, $50 \mathrm{mg} / \mathrm{kg}$ of aztreonam was administered every $8 \mathrm{~h}$ to prevent endogenous colonization by Gram-negative bacteria. We assessed clinical data, pulmonary mechanics and hemodynamic parameters at baseline and $24 \mathrm{~h}$ thereafter, as well as collected tracheal secretions for quantitative microbiology studies (detailed below). In addition, we performed a BAL, as reported below, to quantify bacterial burden at baseline and at 24 and $72 \mathrm{~h}$. Finally, after $76 \mathrm{~h}$, pigs were euthanized, and lung biopsy specimens were taken to quantify bacterial concentration.

Main study. The study was conducted on 10 healthy Large White-Landrace female pigs (mean \pm s.e.m.: $33.7 \pm 1.9 \mathrm{~kg}$; range: $32-37 \mathrm{~kg}$ ).

Animal preparation and mechanical ventilation. The pigs were sedated, orotracheally intubated and connected to a mechanical ventilator (SERVO-I; Maquet). Pigs were initially ventilated in volume-control, square-wave inspiratory flow, duty cycle 0.33 , tidal volume $8 \mathrm{ml} / \mathrm{kg}$, positive end-expiratory pressure of $3-5 \mathrm{~cm} \mathrm{H}_{2} \mathrm{O}$ and respiratory rate adjusted to maintain arterial partial pressure of carbon dioxide within normal range. Inspiratory gases were conditioned via a heated humidifier (MR850; Fisher and Paykel) with a heated inspiratory circuit. The pigs were under continuous anesthesia with a steady infusion of midazolam at $0.2-0.8 \mathrm{mg} \mathrm{kg}^{-1} \mathrm{~h}^{-1}$, propofol at $4-9 \mathrm{mg} \mathrm{kg}^{-1} \mathrm{~h}^{-1}$ and fentanyl at $5-10 \mu \mathrm{gg}^{-1} \mathrm{~h}^{-1}$. Respecting strict sterile technique, we performed an ultrasound-guided femoral artery cannulation for systemic MAP monitoring and blood sample collection. We surgically inserted an 8-French mm catheter into the internal jugular vein (Introflex percutaneous sheath introducer; Edwards Lifesciences) and placed a 7-Fr Swan-Ganz catheter (Swan-Ganz PAC; Edwards Lifesciences) through the introducer. Furthermore, to monitor urinary output throughout the study, we inserted a no. 16 Foley catheter into the bladder via a surgical midline mini-pelvectomy. Throughout the study, $100 \mathrm{mg} / \mathrm{kg}$ of doxycycline every $12 \mathrm{~h}$ and $50 \mathrm{mg} / \mathrm{kg}$ of aztreonam every $8 \mathrm{~h}$ were administered to hinder colonization by endogenous bacteria. After surgical preparation, we placed the pigs in the prone position for bacterial challenge; thereafter, we cyclically moved the pigs into a right- or left-lateral position every $6 \mathrm{~h}$. Throughout the study, we assessed gas exchanges, pulmonary mechanics, hemodynamics, urine output and ventilatory settings every $12 \mathrm{~h}$; we also obtained a complete blood count, basic metabolic panel, liver function, renal panel and coagulation tests.

Bacterial challenge. We prepared a culture of S. pneumoniae (ST276) serotype $19 \mathrm{~A}$, characterized by resistance to penicillin, macrolides and tetracyclines with MICs of ceftriaxone of $1 \mu \mathrm{g} / \mathrm{ml}$, erythromycin $>256 \mu \mathrm{g} / \mathrm{ml}$, azithromycin $>256$ $\mu \mathrm{g} / \mathrm{ml}$, doxycycline $6 \mu \mathrm{g} / \mathrm{ml}$ and levofloxacin $0.75 \mu \mathrm{g} / \mathrm{ml}$. We initially attempted to induce severe pneumococcal pneumonia through single bronchoscopic challenge with $15 \mathrm{~mL}$ of $10^{8}$ c.f.u./ml of S. pneumoniae into each lobe of four pigs set in the prone position: two pigs on the bed oriented horizontally and the other two at an angle above horizontal. After this challenge, the pigs remained healthy and did not develop pneumonia (data not shown). We therefore modified our methods accordingly: shortly after surgical preparation and $4 \mathrm{~h}$ thereafter, two separate slow instillations of $15 \mathrm{ml}$ of $10^{8}$ c.f.u./ml of the aforementioned log-phase culture of S. pneumoniae were performed into each pulmonary lobe of the pigs as they laid on a bed oriented $15^{\circ}$ above horizontal.

Microbiological assessments and clinical definition of pneumonia. Before bacterial challenge and every $24 \mathrm{~h}$ thereafter, we collected tracheal secretions for quantitative microbiology studies. In addition, before bacterial challenge, and after 24 and $72 \mathrm{~h}$, we performed a BAL of the right middle lobe for quantitative microbiology studies and quantification of inflammatory parameters. Finally, before bacterial challenge and every $24 \mathrm{~h}$ thereafter, we assessed blood cultures. At $24 \mathrm{~h}$, clinical diagnosis of pneumonia was confirmed by an association with $S$. pneumoniae colonization $\geq 10^{6}$ c.f.u./ml in tracheal secretions, plus at least one of the following clinical features: body temperature $>38.5^{\circ} \mathrm{C}$ or $<36^{\circ} \mathrm{C}$, a white blood cell count $>14,000$ / $\mathrm{mm}^{3}$ or $<4,000 / \mathrm{mm}^{3}$ and purulent secretions.
Respiratory measurements. We collected respiratory mechanical measurements daily as previously described ${ }^{33}$. Airway pressure was measured proximally to the endotracheal tube, while respiratory flow rates were measured with a heated pneumotachograph. Flow and pressure signals were recorded on a personal computer for subsequent analysis with dedicated software (Colligo; Elekton). The static elastance of the respiratory system was calculated by using standard formulae and applying the rapid occlusion method $^{34}$.

Hemodynamic measurements. After assessment of pulmonary variables, we evaluated hemodynamics and gas exchange (arterial and mixed venous blood) as previously described ${ }^{33}$. Stroke volume, systemic vascular resistance, pulmonary vascular resistance and venous admixture were computed as previously reported ${ }^{33}$. In addition, we computed vasopressor dependency index ${ }^{35}$.

Inflammatory parameters. Before bacterial challenge and at 24,48 and $72 \mathrm{~h}$ thereafter, blood was drawn to measure serum IL-1 $\beta$, IL-6, IL-8, IL-10 and tumor necrosis factor $\alpha$ concentrations. Furthermore, before bacterial challenge and at 24 and $72 \mathrm{~h}$, the aforementioned cytokines were also quantified in BAL. Inflammatory marker concentrations are reported as $\log \mathrm{pg} / \mathrm{liter}$.

Necropsy, post-mortem microbiological and histological studies and the definition of pneumonia. $76 \mathrm{~h}$ after tracheal intubation (72 hours after the initial bacterial challenge), pigs were euthanized. We prematurely discontinued the study in cases of severe refractory respiratory instability (ratio between the partial pressure of arterial oxygen and inspiratory fraction of oxygen $<100$, irrespective of maximal ventilatory support) or refractory hemodynamic instability (MAP $<50 \mathrm{mmHg}$, irrespective of norepinephrine dosing $>0.3 \mathrm{\mu g} \mathrm{kg}^{-1} \mathrm{~min}^{-1}$ ). We collected two samples from the most-affected region of each of the five lobes for histological and quantitative microbiological studies. Lung histology was evaluated according to previously published methods and a six-point injury score $^{36}$. For each lobe, pneumonia was microbiologically confirmed by a quantitative $S$. pneumoniae lobar culture $\geq 10^{3}$ c.f.u./g and histologically confirmed per an injury score $\geq 3$.

Pharmacokinetic analyses. In eight additional pigs, severe pneumococcal pneumonia developed as previously described. After pneumonia was clinically confirmed, different doses of ceftriaxone and levofloxacin were administered to assess pharmacokinetics in the ELF. Initially, we administered $50 \mathrm{mg} / \mathrm{kg}$ of ceftriaxone in two pigs and $10 \mathrm{mg} / \mathrm{kg}$ of levofloxacin in two additional pigs. We sequentially collected plasma and performed BAL in the right middle lobe to measure antibiotic concentrations in the $\mathrm{ELF}^{37}$. Plasma and BAL urea concentrations were determined with a validated enzymatic assay ${ }^{38}$. Our aim was to achieve a concentration of unbound ceftriaxone in the ELF greater than S. pneumoniae MIC for $\geq 14.4 \mathrm{~h}$ ( $60 \%$ of the time between daily dosing $)^{32}$. With respect to levofloxacin, our aim was to achieve a ratio between the area under the concentration-time curve from 0 to $24 \mathrm{~h}$ post-dose of bound levofloxacin and S. pneumoniae MIC $>40^{39}$.

Statistical analysis. Continuous variables were described as means and standard deviations or standard errors and median (25th-75th quartiles). Categorical variables were described as frequencies and percentages. Continuous variables were analyzed by using an REML analysis, based on repeated measures approach (PROC MIXED) and including times of assessment and pulmonary lobes as factors. A compound of symmetry or univariate (co)variance structure was used to model the within-subjects' errors. For each continuous variable, the overall $F$ test was first assessed for significance $(P \leq 0.05)$. Each pair-wise comparison was also performed, corrected by using the Bonferroni test to control for the experiment-wise error rate, and considered significant if its $P$ value was $\leq 0.05$. We tested the assumption in PROC MIXED with respect to normality of the model residuals. In cases of non-normally distributed residuals, we used the Friedman test. Categorical variables were analyzed by using Fisher's exact test and the Cochran-Mantel-Haenszel test. All statistical analyses were performed by using SAS software (version 9.4; SAS Institute).

Reporting Summary. Further information on research design is available in the Nature Research Reporting Summary linked to this article.

\section{Data availability}

The datasets generated during and/or analyzed during the current study are available from the corresponding author on reasonable request.

\section{References}

33. Li Bassi, G. et al. Effects of duty cycle and positive end-expiratory pressure on mucus clearance during mechanical ventilation*. Crit. Care Med. 40, 895-902 (2012)

34. D'Angelo, E. et al. Respiratory mechanics in anesthetized paralyzed humans: effects of flow, volume, and time. J. Appl. Physiol. (1985) 67, 2556-2564 (1989). 
35. Cruz, D. N. et al. Early use of polymyxin B hemoperfusion in abdominal septic shock: the EUPHAS randomized controlled trial. JAMA 301, 2445-2452 (2009).

36. Li Bassi, G. et al. Gravity predominates over ventilatory pattern in the prevention of ventilator-associated pneumonia. Crit. Care Med. $\mathbf{4 2}$, e620-e627 (2014).

37. Onyeji, C. O. et al. Comparative efficacies of levofloxacin and ciprofloxacin against Streptococcus pneumoniae in a mouse model of experimental septicaemia. Int. J. Antimicrob. Agents 12, 107-114 (1999).

38. Rennard, S. I. et al. Estimation of volume of epithelial lining fluid recovered by lavage using urea as marker of dilution. J. Appl. Physiol. (1985) 60, 532-538 (1986).

39. Preston, S. L. et al. Pharmacodynamics of levofloxacin: a new paradigm for early clinical trials. JAMA 279, 125-129 (1998).

\section{Acknowledgements}

We deeply thank Francesc Marco (Microbiology Department, Hospital Clinic) for his swift and valuable support in identifying and providing human S. pneumoniae strains upon study commencement. In addition, we acknowledge the healthcare providers from the pulmonary intensive care unit at Hospital Clinic, Barcelona for all their encouragement during these studies. Support was provided by the Instituto de Salud Carlos III de Madrid (Spain) (FIS PI15/00506), Fundació La Marató de TV3 (201831-10), the Institut d'Investigacions Biomèdiques August Pi i Sunyer (IDIBAPS), Centro de Investigación Biomedica En Red- Enfermedades Respiratorias (CIBERES) and BITRECS (IDIBAPS) 2019.

\section{Author contributions}

R.A., G.L.B, A.M. and A.T. participated in the development of the protocol, study design, study management, statistical analysis and data interpretation and wrote the first draft of the report. L.F.B, E.A.X., M. Rigol, G.F., C.T., J.B., F.P., M.C., T.C., C.C., M.Y., H.Y., M.A., J.D.M., F.D.R., M.A.S., M. Rinaudo and S.T. participated in data collection and interpretation and critically reviewed the first draft of the report. M.J.S., D.P.N., A.A. and J.R. participated in study design and reviewed the report.

\section{Competing interests}

A.T. has received grants from MedImmune, Cubist, Bayer, Theravance and Polyphor and personal fees as an advisory board member from Bayer, Roche, The Medicines CO and Curetis. He has received personal fees as a member of the speaker's bureau from GSK, Pfizer, Astra Zeneca and Biotest Advisory Board, outside the submitted work. None of the other authors have any competing interests with the current publication.

\section{Additional information}

Supplementary information The online version contains supplementary material available at https://doi.org/10.1038/s41684-021-00876-y.

Correspondence and requests for materials should be addressed to Antoni Torres. Peer review information Lab Animal thanks the anonymous reviewer(s) for their contribution to the peer review of this work.

Reprints and permissions information is available at www.nature.com/reprints. 


\section{nature portfolio}

Corresponding author(s): LABAN-A00841

Last updated by author(s): Aug 5, 2021

\section{Reporting Summary}

Nature Portfolio wishes to improve the reproducibility of the work that we publish. This form provides structure for consistency and transparency in reporting. For further information on Nature Portfolio policies, see our Editorial Policies and the Editorial Policy Checklist.

\section{Statistics}

For all statistical analyses, confirm that the following items are present in the figure legend, table legend, main text, or Methods section.

$\mathrm{n} / \mathrm{a}$ Confirmed

\ The exact sample size $(n)$ for each experimental group/condition, given as a discrete number and unit of measurement

$\bigotimes$ A statement on whether measurements were taken from distinct samples or whether the same sample was measured repeatedly

$\triangle$ The statistical test(s) used AND whether they are one- or two-sided

Only common tests should be described solely by name; describe more complex techniques in the Methods section.

$\bigotimes$ A description of all covariates tested

\A description of any assumptions or corrections, such as tests of normality and adjustment for multiple comparisons

$\triangle$ A full description of the statistical parameters including central tendency (e.g. means) or other basic estimates (e.g. regression coefficient)

AND variation (e.g. standard deviation) or associated estimates of uncertainty (e.g. confidence intervals)

For null hypothesis testing, the test statistic (e.g. $F, t, r$ ) with confidence intervals, effect sizes, degrees of freedom and $P$ value noted

Give $P$ values as exact values whenever suitable.

Х $\square$ For Bayesian analysis, information on the choice of priors and Markov chain Monte Carlo settings

$\bigotimes \square$ For hierarchical and complex designs, identification of the appropriate level for tests and full reporting of outcomes

$\triangle \square$ Estimates of effect sizes (e.g. Cohen's d, Pearson's $r$ ), indicating how they were calculated

Our web collection on statistics for biologists contains articles on many of the points above.

\section{Software and code}

Policy information about availability of computer code

Data collection Microsoft Excel for Mac (version 16.47.1; Redmond, WA, USA).

Data analysis SAS software (version 9.4; SAS Institute, Cary, NC, USA).

For manuscripts utilizing custom algorithms or software that are central to the research but not yet described in published literature, software must be made available to editors and

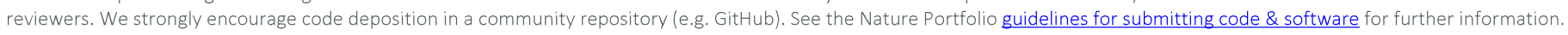

\section{Data}

Policy information about availability of data

All manuscripts must include a data availability statement. This statement should provide the following information, where applicable:

- Accession codes, unique identifiers, or web links for publicly available datasets

- A description of any restrictions on data availability

- For clinical datasets or third party data, please ensure that the statement adheres to our policy

The datasets generated during and/or analysed during the current study are available from the corresponding author on reasonable request. 
Please select the one below that is the best fit for your research. If you are not sure, read the appropriate sections before making your selection.

\ Life sciences

Behavioural \& social sciences

Ecological, evolutionary \& environmental sciences

For a reference copy of the document with all sections, see nature.com/documents/nr-reporting-summary-flat.pdf

\section{Life sciences study design}

All studies must disclose on these points even when the disclosure is negative.

\begin{tabular}{l|l} 
Sample size & No sample size was performed as we were developing a novel model. Nevertheless, we have used published pneumonia large animal models \\
to determine the final sample size (Li Bassi G., et Anesthesiology 2014; Reyes L., et al. PLoS One 2016; Martinez-Olondris P. et al, Eur Respir J
\end{tabular} 2010).

Data exclusions No data were excluded from the analyses

Replication Clinical measures and samples were taken at baseline and throughout the experiment to ensure replication. No significant standard deviations were found between animals in each group. Also, differences at baseline were analyzed between both groups (infected and control), and we did not found any.

Randomization Randomization was not relevant in our study, as we are here describing a novel model.

Blinding Randomization was not relevant in our study, as we are here describing a novel model.

\section{Reporting for specific materials, systems and methods}

We require information from authors about some types of materials, experimental systems and methods used in many studies. Here, indicate whether each material, system or method listed is relevant to your study. If you are not sure if a list item applies to your research, read the appropriate section before selecting a response.

Materials \& experimental systems

$\mathrm{n} / \mathrm{a}$ Involved in the study

\ $\square$ Antibodies

Methods

$\bigotimes \square$ Eukaryotic cell lines

Х Palaeontology and archaeology

$\mathrm{n} / \mathrm{a}$ Involved in the study

X $\square$ ChIP-seq

Х Flow cytometry

$\square \bigotimes$ Animals and other organisms

Х $\square$ MRI-based neuroimaging

$\bigotimes \square$ Human research participants

\ $\square$ Clinical data

$\bigotimes \square$ Dual use research of concern

\section{Animals and other organisms}

Policy information about studies involving animals; ARRIVE guidelines recommended for reporting animal research

Laboratory animals

Wild animals

Field-collected samples

Ethics oversight

\section{Large-White Landrace female pigs}

The study did not involve wild animals

The study did not involve samples collected from the field

Animal Experimentation Ethics Committee of the University of Barcelona (approval reference number: DAAM 42/12). 